\title{
Sulforaphane downregulated fatty acid synthase and inhibited microtubule-mediated mitophagy leading to apoptosis
}

\author{
Yuting Yan ${ }^{1,2,3}$, Yan Zhou ${ }^{1,2}$, Juntao $\mathrm{Li}^{1,2}$, Zhongnan Zheng ${ }^{1,2}$, Yabin $\mathrm{Hu}^{1,2}$, Lei $\mathrm{Li}^{4,5}$ and Wei Wu (D) ${ }^{1,2}$ \\ (c) The Author(s) 2021
}

We previously demonstrated that sulforaphane (SFN) inhibited autophagy leading to apoptosis in human non-small cell lung cancer (NSCLC) cells, but the underlying subcellular mechanisms were unknown. Hereby, high-performance liquid chromatography-tandem mass spectrometry uncovered that SFN regulated the production of lipoproteins, and microtubule- and autophagy-associated proteins. Further, highly expressed fatty acid synthase (FASN) contributed to cancer malignancy and poor prognosis. Results showed that SFN depolymerized microtubules, downregulated FASN, and decreased its binding to a-tubulin; SFN downregulated FASN, acetyl CoA carboxylase (ACACA), and ATP citrate lyase (ACLY) via activating proteasomes and downregulating transcriptional factor SREBP1; SFN inhibited the interactions among a-tubulin and FASN, ACACA, and ACLY; SFN decreased the amount of intracellular fatty acid (FA) and mitochondrial phospholipids; and knockdown of FASN decreased mitochondrial membrane potential $(\Delta \Psi \mathrm{m})$ and increased reactive oxygen species, mitochondrial abnormality, and apoptosis. Further, SFN downregulated mitophagy-associated proteins Bnip3 and NIX, and upregulated mitochondrial LC3 II/I. Transmission electron microscopy showed mitochondrial abnormality and accumulation of mitophagosomes in response to SFN. Combined with mitophagy inducer CCCP or autophagosome-lysosome fusion inhibitor Bafilomycin A1, we found that SFN inhibited mitophagosome-lysosome fusion leading to mitophagosome accumulation. SFN reduced the interaction between NIX and LC3 II/I, and reversed CCCP-caused FA increase. Furthermore, knockdown of a-tubulin downregulated NIX and BNIP3 production, and upregulated LC3 II/l. Besides, SFN reduced the interaction and colocalization between a-tubulin and NIX. Thus, SFN might cause apoptosis via inhibiting microtubule-mediated mitophagy. These results might give us a new insight into the mechanisms of SFNcaused apoptosis in the subcellular level.

Cell Death and Disease (2021)12:917; https://doi.org/10.1038/s41419-021-04198-2

\section{INTRODUCTION}

We reported that sulforaphane (SFN) metabolite-induced microtubule disruption contributed to the inhibition of cell proliferation and autophagy leading to apoptosis [1, 2]. Meanwhile, SFN might cause swollen mitochondria in non-small cell lung cancer (NSCLC) [3]. In a separate study, we found that SFN might inhibit mitophagy and mitochondrial glucose metabolism via impeding the trafficking and translocation of damaged proteins into mitochondria (unpublished data). Hence, some deeper mechanisms involved in SFN-triggered mitochondrial dysfunction and metabolic abnormality might result in apoptosis. Besides, report showed that abnormal lipid metabolism might cause cell apoptosis [4]. De novo synthesis is the main pathway for cancer cells to produce fatty acids (FAs); FA synthase (FASN) is highly expressed in various cancers including lung cancer and low production of FASN in NSCLC patients have longer survival [5-7]. Study showed that downregulation of FASN production caused apoptosis in tumor cells rather than normal cells [8]. FAs are essential components of biofilm lipids and are important substrates for energy metabolism [9-11]; tumor cells require large amounts of FAs for rapid division and proliferation [12, 13].
Glucose uptake by tumor cells produces pyruvate by glycolysis, a small part of pyruvate molecules enter the mitochondria to undergo oxidative decarboxylation to form citric acids. Citric acids were transported out of mitochondria and catalyzed by ATP citrate lyase (ACLY) to release acetyl CoA. Acetyl CoA carboxylase (ACACA) catalyzes acetyl CoA to form malonyl CoA. In the presence of nicotinamide adenine dinucleotide phosphate, acetyl CoA interacts with malonyl CoA to synthesize FAs by FASN [14]. FA metabolites might act as signal molecules involved in tumor growth or participate in protein modification after translation [8]. Therefore, the interference of FA synthesis by inhibiting FA synthesis-related molecules might help us establish an effective anti-cancer therapy.

Studies showed that cruciferous plant-derived SFN inhibited lipid production and induced apoptosis in prostate cancer cells $[13,15]$. SFN induced apoptosis of NSCLC cells in a concentration-dependent manner at $20 \mu \mathrm{M}$ concentration [3]. Just recently, we reported that the combination of SFN metabolites and paclitaxel reduced the dosage and restored sensitivity of paclitaxel-resistant cells to paclitaxel [16]. We also

\footnotetext{
${ }^{1}$ Department of Biochemistry and Molecular Biology, School of Basic Medical Sciences, Capital Medical University, Beijing, China. ${ }^{2}$ Beijing Key Laboratory for Tumor Invasion and Metastasis, Beijing, China. ${ }^{3}$ State Key Laboratory of Cardiovascular Disease, Fuwai Hospital, Beijing 100037, China. ${ }^{4}$ Central Laboratory, Capital Medical University, Beijing, China. ${ }^{5}$ Capital Medical University, No. 10, Xitoutiao, Beijing 100069, China. ${ }^{凶}$ email: weiwu207@ccmu.edu.cn Edited by Professor Quan Chen
} 


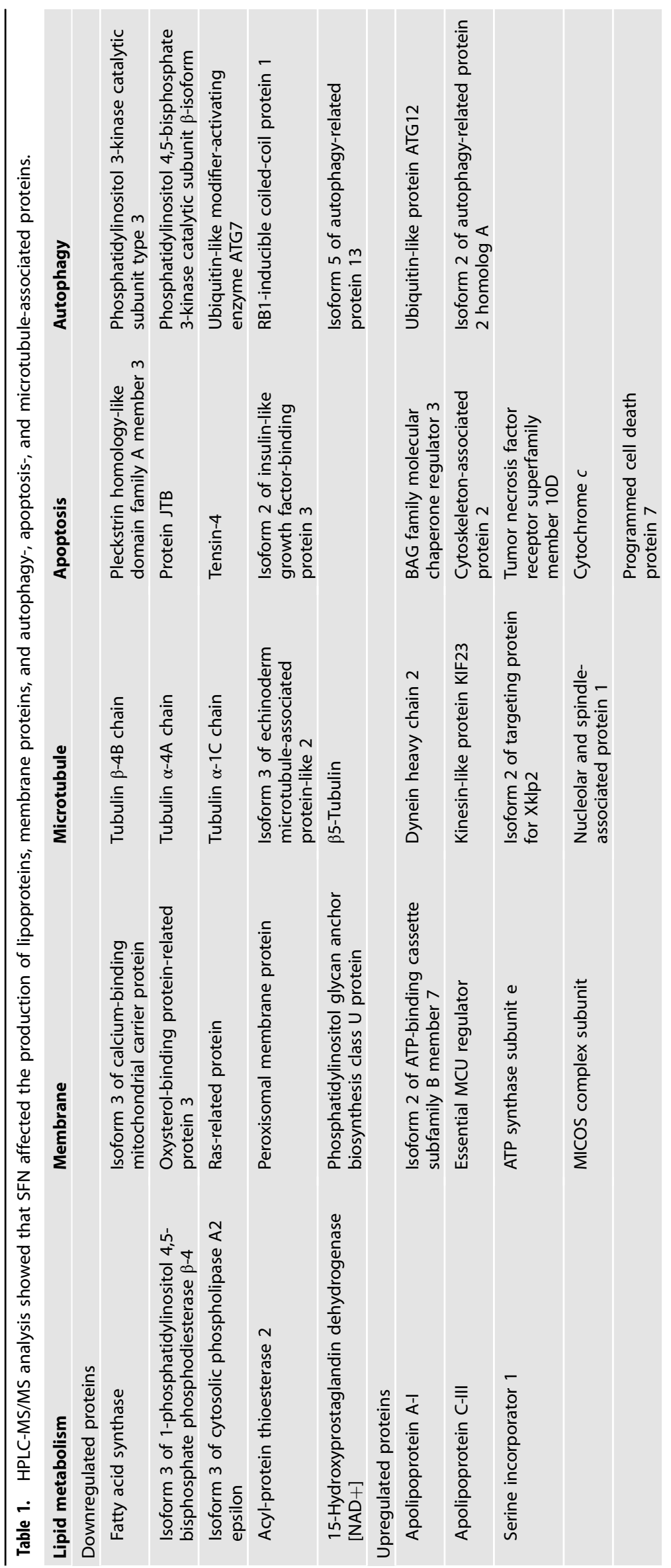


A

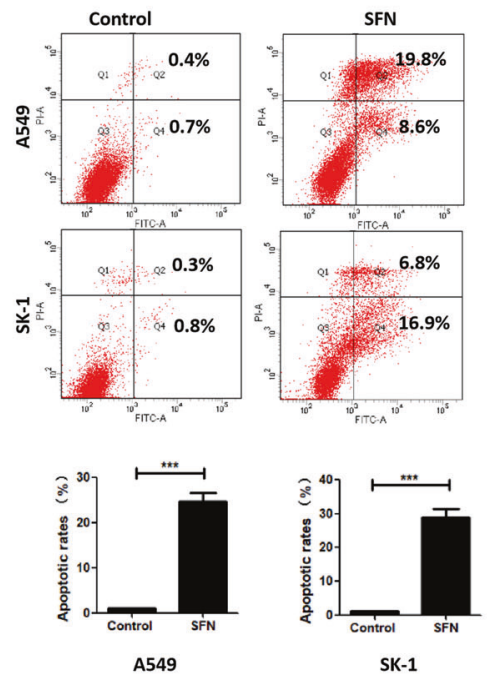

E

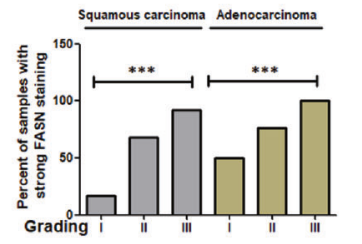

$\mathbf{F}$

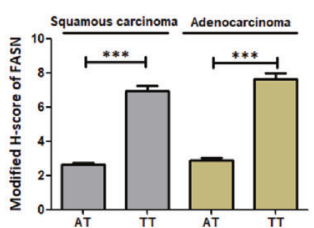

B

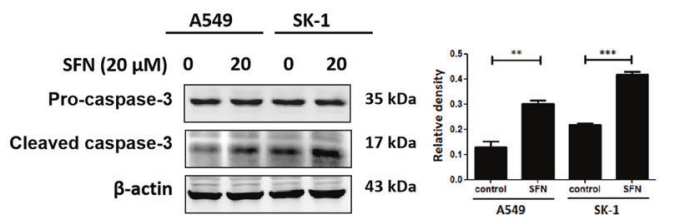

C

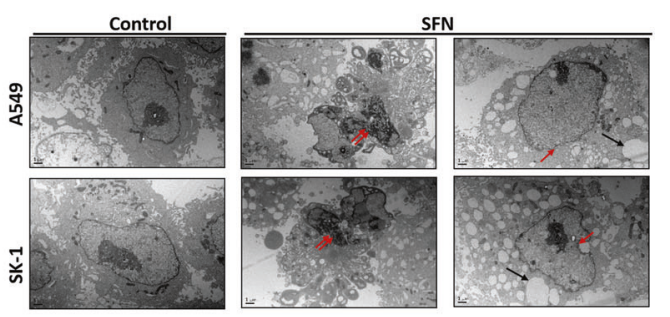

D

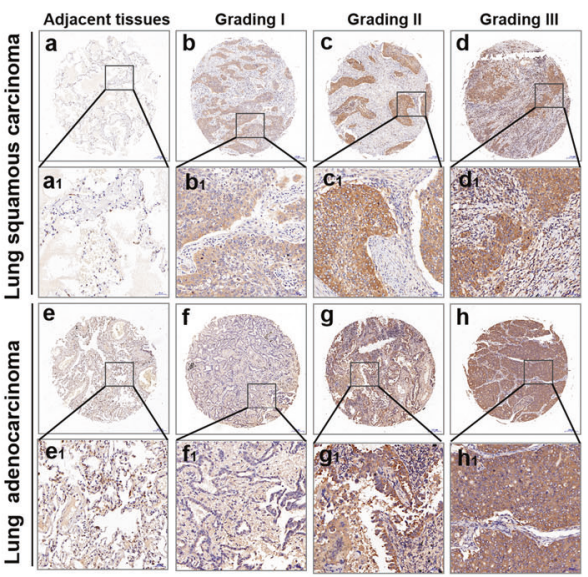

Fig. 1 SFN-induced apoptosis might result from the inhibition of microtubule-mediated FA synthesis. A Both of A549 and SK-1 cells were treated with SFN $(20 \mu \mathrm{M})$ for $24 \mathrm{~h}$, then the cells were collected and the percentages of cell apoptosis were analyzed by a flow cytometer. B Caspase-3 proteins were analyzed via western blotting in A549 and SK- 1 cells treated with SFN (20 $\mu$ M). C After induction with $20 \mu \mathrm{M}$ SFN, both A549 and SK-1 cells were collected and fixed. Cell pellets were cut into thin slices and stained with uranium acetate and lead nitrate. Cell morphology was viewed with TEM. Double red arrows marked nuclear fragments, black arrows indicate sporadic vacuoles, and red arrows indicate membrane disruption. Scale bar: $1 \mu \mathrm{m}$. D Levels of FASN were assessed in the microarray tissues of lung adenocarcinoma and lung squamous carcinoma. FASN levels in the 150 adjacent tissues were tested as the controls (A, E) and 150 cancer tissues with three malignant grades (B-D, F-H) were used. Upper panel: magnification $\times 50$, scale bars: $500 \mu \mathrm{m}$. Lower panel: magnification $\times 200$, scale bars: $100 \mu \mathrm{m}$. E Percentages of samples with strong FASN staining in 75 tumor tissues, which were divided into three malignant grades. $\mathbf{F}$ The comparison of $\mathrm{H}$ scores for FASN accumulation between adjacent and tumor tissues were done in lung adenocarcinoma and lung squamous carcinoma (AT: 75 adjacent tissues; TT: 75 tumor tissues). Data were shown as means \pm SEM $(n \geq 3)$. ${ }^{* *} P \leq 0.001 ;{ }^{* *} P \leq 0.0001$.

found that SFN metabolites induced apoptosis by inhibiting microtubule-mediated autophagy in NSCLC [1]. Microtubules are the dimers formed by a-tubulin, $\beta$-tubulin, and a small part of microtubule-binding proteins, such as microtubule-associated protein 1 light chain 3 (LC3 II/I) and stathmin-1 [17]. However, it was not clear whether SFN-caused microtubule disruption contributed to inhibition of FA synthesis and mitophagy. In particular, whether the inhibition of mitophagy regulated FA production leading to apoptosis was unknown.

Bafilomycin A1 (Baf-A1) is an autophagy inhibitor that blocks the fusion of autophagosome to lysosome. We previously reported that, combined with Baf-A1, SFN metabolites inhibited the fusion of autophagosome to lysosome and caused the accumulated autophagy flux LC3 II/I leading to apoptosis [1]. LC3 II/I located on the autophagic membrane and were autophagy marker proteins [18]. Mitophagy helps cells to remove damaged mitochondria and maintain cellular proteostasis $[19,20]$. Mitophagy is mainly mediated by the PINK/Parkin, BNIP3/NIX, and FUNDC1 signaling pathways. NIX is a NIP3 protein on mitochondria in the $\mathrm{Bcl}-2$ family. BNIP3 is a member of the $\mathrm{Bcl}-2$ protein family and belongs to the $\mathrm{BH} 3$ subfamily containing only the $\mathrm{BH} 3$ domain $[21,22]$. The damaged mitochondria are surrounded by a bilayer membrane to form mitophagosomes. NIX on the outer membrane of mitochondria recruits LC3 II/I to form autophagosomes enveloping damaged mitochondria; the phagocytosis of damaged mitochondria maintains the homeostasis of cells $[23,24]$. Consequently, SFN might inhibit the fusion of mitophagosomes to lysosomes and the recruitment of LC3 II/I to NIX [25].

Taken together, we hypothesized that SFN inhibited microtubule-mediated FA synthesis disrupting mitochondrial membrane structures and inhibited microtubule-mediated mitophagy leading to apoptosis. These studies might help us understand the deeper mechanisms that SFN produced in the subcellular levels and establish efficient anti-cancer therapies.

\section{RESULTS}

SFN-caused apoptosis might result from the downregulation of FA synthesis-associated proteins and microtubuleassociated proteins

These results by high-performance liquid chromatographytandem mass spectrometry (HPLC-MS/MS) analysis showed that 

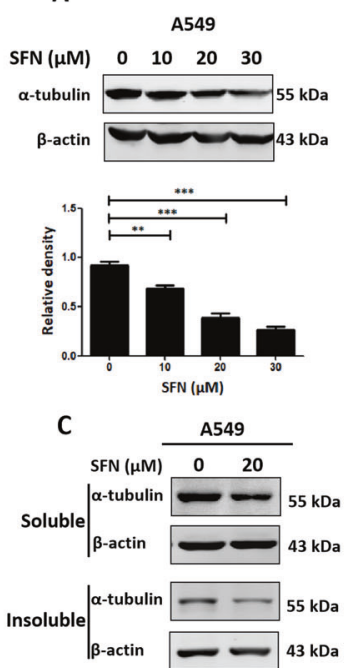

D

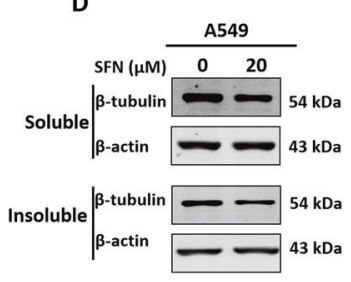

E

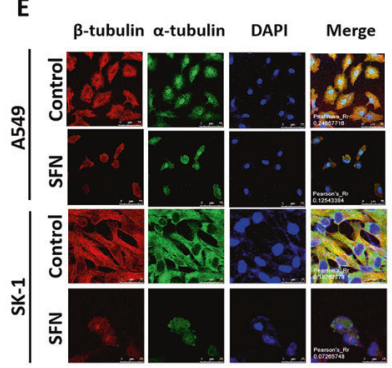

Merge
B
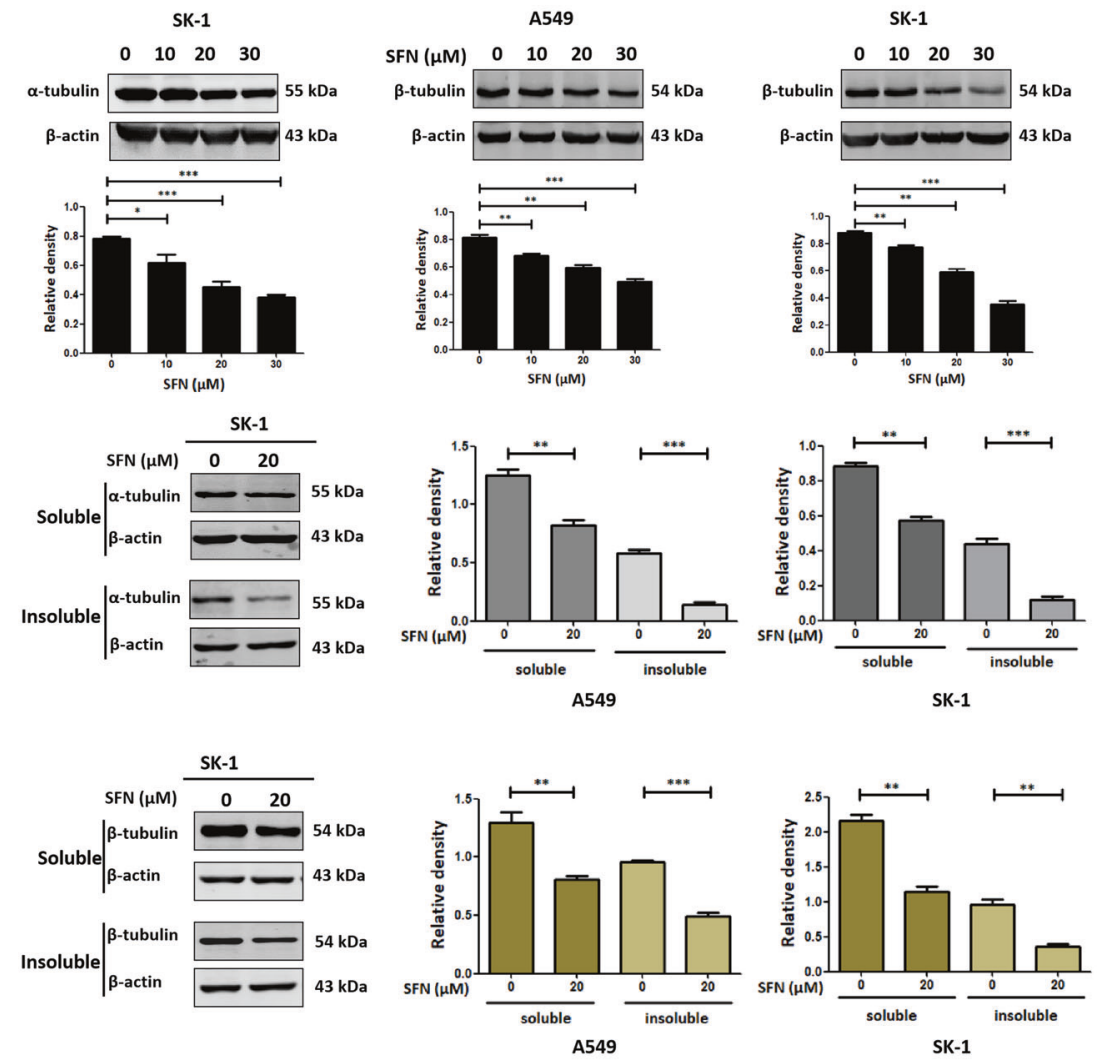

Fig. 2 SFN caused microtubule disruption by breaking a-tubulin and $\boldsymbol{\beta}$-tubulin dimer. A, B The accumulation of $\alpha$-tubulin and $\beta$-tubulin was detected by western blotting after the treatment of 0, 10, 20, and $30 \mu \mathrm{M}$ SFN for $24 \mathrm{~h}$ in A549 and SK-1 cells. C, D Both A549 and SK-1 cells were treated with/without $20 \mu \mathrm{M}$ SFN for $24 \mathrm{~h}$. Then the accumulation of soluble and insoluble $\alpha$-tubulin (C) or $\beta$-tubulin (D) was determined by western blotting in both A549 and SK-1 cells. E Immunofluorescence and confocal microscopy were employed to view the colocalization of $\alpha$-tubulin to $\beta$-tubulin. Scale bar: A549: $75 \mu \mathrm{m}$, SK-1: $25 \mu \mathrm{m}$. Data were shown as means \pm SEM $(n \geq 3)$. ${ }^{* *} P \leq 0.001 ;{ }^{* * *} P \leq 0.0001$.

SFN affected the production of lipoproteins, membrane proteins, and autophagy-, apoptosis- and microtubule-associated proteins (Table 1). Further, the rates of apoptosis were increased and cleaved caspase-3 level was increased after $24 \mathrm{~h}$ treatment with SFN (Fig. 1A, B). Cell apoptotic features, such as chromatin condensation, apoptotic bodies, and vacuolization were viewed by a transmission electron microscope (TEM) (Fig. 1C). Tissue microarray analysis showed a positive correlation of FASN level to the malignancy of NSCLC (Fig. 1D). FASN level is positively correlated to the malignant grades of tumor cells in both lung squamous cell carcinoma and lung adenocarcinoma (Fig. 1E). The correlation of FASN expression to pathological grading of NSCLC was analyzed by calculating the $\mathrm{H}$-score (Table 2). The level of FASN in tumor tissues is higher than that in adjacent tissues (Fig. 1F). These results suggested that SFN-caused apoptosis might result from the downregulation of $F A$ synthesis-associated proteins and the dysfunction of microtubule- and autophagy-associated proteins, and membrane proteins.
SFN inhibited the polymerization of microtubules

Cells were treated with different concentrations of SFN for $24 \mathrm{~h}$. Results showed that SFN significantly downregulated a-tubulin in a concentration-dependent manner (Fig. 2A). Meanwhile, $\beta$-tubulin was also downregulated in a concentration-dependent manner (Fig. 2B). Microtubule polymerization assays showed that the amounts of both soluble and insoluble a-tubulin or $\beta$-tubulin were lower vs. control, indicating that the SFN depolymerized the microtubules (Fig. 2C, D). Immunofluorescence staining showed that SFN disrupted microtubules and reduced the colocalization of a-tubulin to $\beta$-tubulin (Fig. 2E). In a separate study, we found that SFN reduced the interaction between $a$-tubulin and $\beta$-tubulin (unpublished data). These results indicated that SFN inhibited microtubule polymerization leading to microtubule disruption.

SFN inhibited microtubule-mediated FA synthesis via activating $26 \mathrm{~S}$ proteasome

After treatment with gradient concentrations of SFN for $24 \mathrm{~h}$, the results showed that SREBP1, FASN, ACACA, and ACLY were 
Table 2. Correlation of FASN expression to clinicopathological characteristics of lung cancer patients.

\begin{tabular}{|c|c|c|c|c|c|c|c|c|c|}
\hline \multirow[t]{2}{*}{ Variable } & \multicolumn{4}{|c|}{ Lung squamous carcinoma } & \multirow[t]{2}{*}{ Variable } & \multicolumn{4}{|c|}{ Lung adenocarcinoma } \\
\hline & All patients & Low & High & $P$-value & & All patients & Low & High & $P$-value \\
\hline Gender & & & & 0.458 & Gender & & & & 0.567 \\
\hline Female & 4 & 2 & 2 & & Female & 35 & 7 & 28 & \\
\hline Age (years) & & & & 0.893 & Age (years) & & & & 0.136 \\
\hline$>60$ & 40 & 12 & 28 & & $>60$ & 41 & 12 & 29 & \\
\hline Differentiation & & & & $0.001^{*}$ & Differentiation & & & & $0.003^{*}$ \\
\hline 1 & 6 & 5 & 1 & & 1 & 14 & 7 & 7 & \\
\hline II & 44 & 14 & 30 & & II & 42 & 10 & 32 & \\
\hline III & 25 & 2 & 23 & & III & 19 & 0 & 19 & \\
\hline Staging & & & & 0.144 & Staging & & & & 0.838 \\
\hline IIB & 10 & 3 & 7 & & IIB & 4 & 1 & 3 & \\
\hline IIIA & 22 & 6 & 16 & & IIIA & 11 & 4 & 7 & \\
\hline IIIB & 2 & 0 & 5 & & IIIB & 3 & 0 & 3 & \\
\hline IV & 5 & 0 & 5 & & III & 2 & 0 & 2 & \\
\hline & & & & & IV & 4 & 1 & 3 & \\
\hline
\end{tabular}

Low (Score 0-4), High (Score 5-12).

${ }^{*} p<0.05$ was defined as statistically significant.

downregulated in a concentration-dependent manner (Fig. 3A). Combined with proteasome inhibitor PS-341, the results show that SFN degraded SREBP1, FASN, ACACA, and ACLY via activating 26S proteasome (Fig. 3B). After knockdown of SREBP1 production, the FA-related proteins ACACA, FASN, and ACLY were downregulated (Fig. 3C). These results showed that SFN degraded the key enzymes in FA synthesis through two pathways, the 26Sproteasomal pathway and the downregulation of SREBP1, as a transcriptional factor for FASN production. Correlation analysis showed that level of SREBP1 had a correlation to FASN level and the level of either ACACA or ACLY, or FASN was closely correlated to FA synthesis (Fig. 3D). Moreover, we found that SFN inhibited the interaction between a-tubulin and FASN, ACACA, and ACLY (Fig. 3E); meanwhile, SFN lowered the colocalization of FASN, ACACA, and ACLY to a-tubulin (Fig. 3F). After knockdown of $\beta$-tubulin production, the FA-related proteins ACACA, FASN, and $A C L Y$ was downregulated (Fig. $3 G$ ). These results showed that disrupted microtubule led to decrease of expression level of FArelated proteins. The levels of FAs and mitochondrial phospholipids were significantly reduced in response to SFN (Fig. $3 \mathrm{H}, \mathrm{I}$ ). Therefore, SFN might lower the production of mitochondrial phospholipids by inhibiting microtubule-mediated FA synthesis leading to the dysfunction of mitochondrial membranes.

\section{SFN-triggered downregulation of FASN level contributed to apoptosis}

The FASN level was successfully knocked down via small interfering RNA (siRNA) interference leading to the elevated intracellular reactive oxygen species (ROS) level and downregulation of mitochondrial membrane potential $\left(\Delta \Psi_{\mathrm{m}}\right)$ (Fig. $\left.4 \mathrm{~A}-\mathrm{C}\right)$. Abnormal cell membrane structures, swollen mitochondria, and apoptotic features were viewed (Fig. 4D). Knockdown of FASN production induced cell apoptosis (Fig. 4E). The levels of FA and mitochondrial phospholipid were significantly reduced in the FASN siRNA group vs. control (Fig. 4F, G). After knockdown of
FASN production, the microtubule-associated protein LC3 II/I was upregulated (Fig. $4 \mathrm{H}$ ). These results indicated that inhibition of FASN production might reduce cellular FA content, thereby reducing intracellular phospholipid level, leading to mitochondrial membrane instability and apoptosis.

\section{SFN inhibited microtubule-mediated mitophagy and the fusion of mitophagosomes to lysosomes}

We found that mitophagy-associated protein BNIP3 localized to mitochondria and SFN downregulated BNIP3 level (Fig. 5A), and the mitochondrial mitophagy-associated protein NIX was expressed in both the mitochondria and cytoplasm, and the expressions were inhibited by SFN in a concentration gradient (Fig. 5B, C). The accumulated microtubule-associated protein LC3 II by SFN was mainly present in mitochondria and SFN treatment had no significant effect on LC3 II/I in cytoplasm (Fig. 5D, E). Mitophagosomes in the cells were viewed after treated with SFN, whereas autolysosome was rarely visible (Fig. 5F). After co-treated with the mitophagy inducer carbonylcyanide 3-chlorophenylhydrazone (CCCP), SFN reversed LC3 II/I downregulation (Fig. 5G, H). After cotreated with Baf-A1, SFN did not make a significant change in LC3 II/I level (Fig. 5I, J). These results indicated that SFN inhibited mitophagy by hindering the fusion of mitophagosomes to lysosomes. Coimmunoprecipitation showed that SFN reduced the interaction between NIX and LC3 II/I in the cytoplasm and mitochondria, and inhibited the initiation of mitophagy (Fig. 5K, L). These results showed that SFN inhibited mitophagy by lowering the expression of mitophagy-associated proteins and the interaction between microtubule-associated protein LC3 II/I and mitophagy-associated proteins inhibiting the degradation of mitophagosomes.

SFN induced apoptosis by inhibiting microtubule-mediated FA synthesis and mitophagy

After the cells were treated with the mitophagy inducer CCCP, FA levels were increased. Combined with SFN, the increased FA 

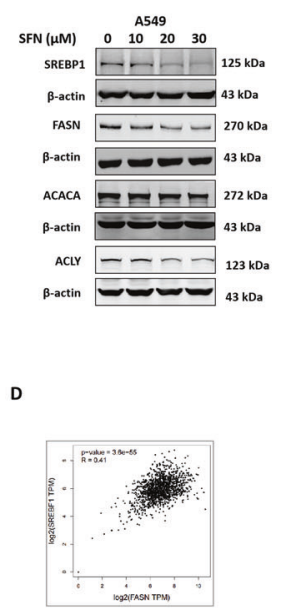

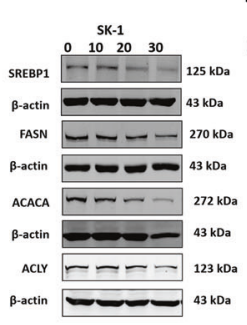

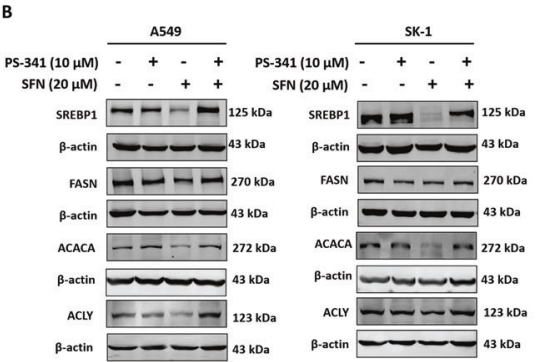

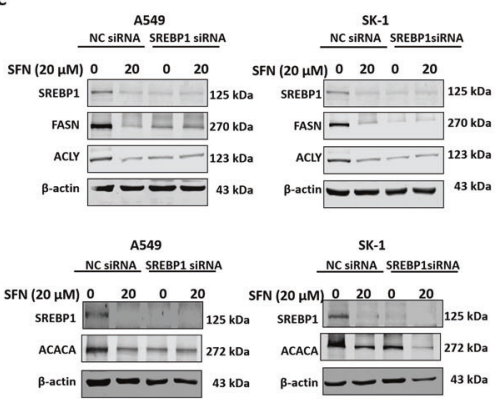

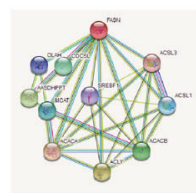
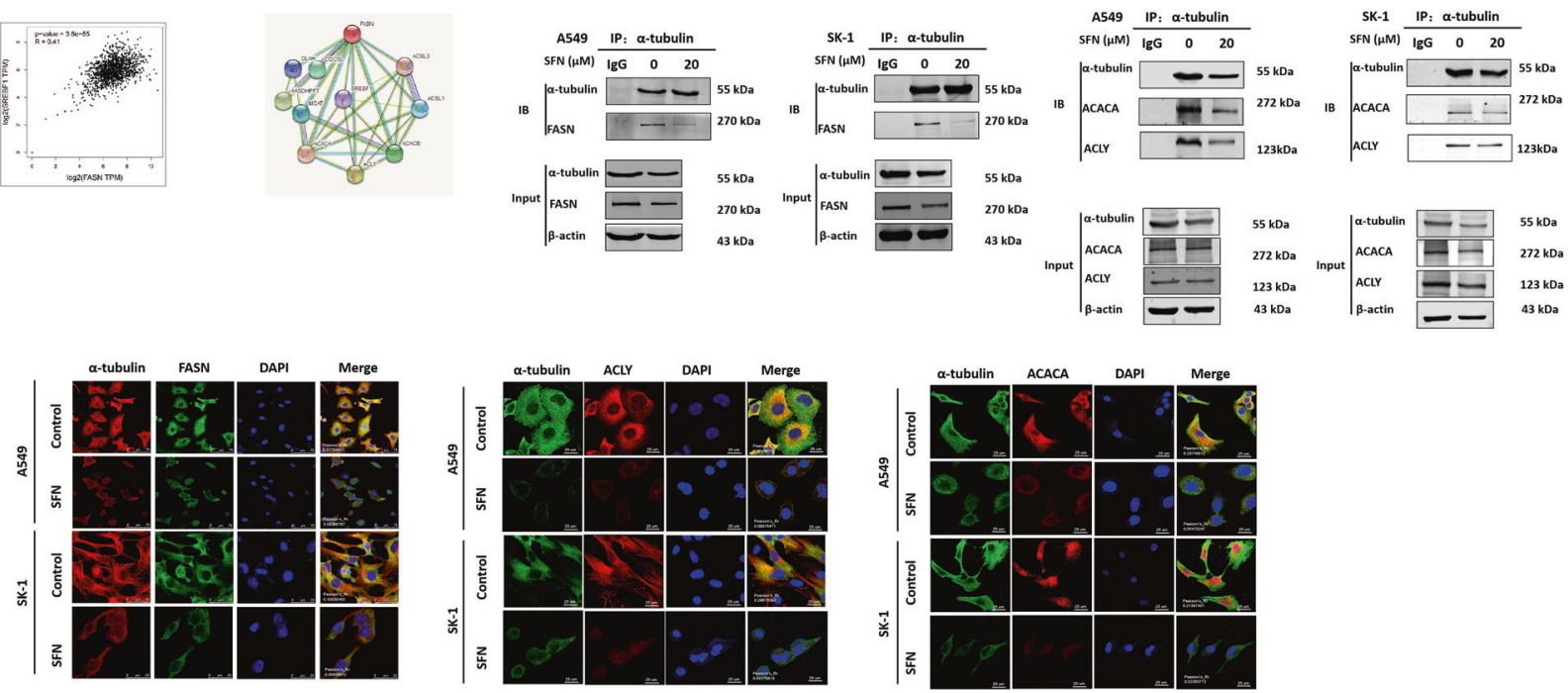

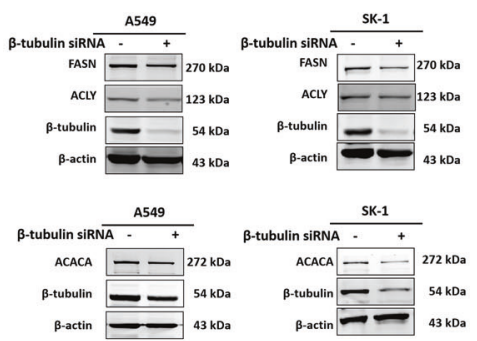

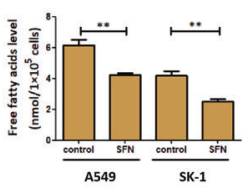

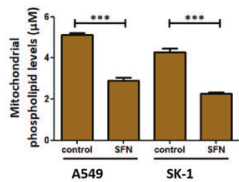

Fig. 3 SFN inhibited microtubule-mediated FA synthesis via activating 265 proteasome. A Both A549 and SK-1 cells were treated with 0 , 10,20 , and $30 \mu \mathrm{M}$ SFN for $24 \mathrm{~h}$. Western blotting was used to analyze the accumulation of SREBP1, FASN, ACACA, and ACLY. B Both A549 and SK-1 cells were pretreated with PS-341 $(10 \mu \mathrm{M})$ for $2 \mathrm{~h}$, then treated with SFN $(20 \mu \mathrm{M})$ for $24 \mathrm{~h}$. The accumulation of SREBP1, FASN, ACACA, and ACLY was analyzed by western blotting. C After knockdown of SREBP1, western blotting was used to analyze ACACA, FASN, and ACLY accumulation. D GEPIA database was used to analyze the correlation between transcription factor SREBP1 and FASN. If R closer is to "1," the correlation between the two proteins is higher. Via the String net, we analyzed the interactions among FASN and related partners. E After induction with $20 \mu \mathrm{M}$ SFN for $24 \mathrm{~h}$, coimmunoprecipitation was employed to detect the interaction between $\alpha$-tubulin and FASN, ACACA, and ACLY. F Immunofluorescence and confocal microscopy were employed to observe the colocalization of $\alpha$-tubulin to FASN (scale bar: A549: $75 \mu \mathrm{m}$, SK-1: $25 \mu \mathrm{m}$ ), ACACA (scale bar: $25 \mu \mathrm{m}$ ), ACLY (scale bar: $25 \mu \mathrm{m}$ ). G After knockdown of $\beta$-tubulin, western blotting was used to analyze ACACA, FASN, and ACLY accumulation. $H$ Determination of total free FA content after induction with $20 \mu \mathrm{M}$ SFN for $24 \mathrm{~h}$. I After induction with $20 \mu \mathrm{M}$ SFN for $24 \mathrm{~h}$, mitochondrial phospholipid content was detected by kit. Data were shown as means \pm SEM $(n \geq 3) .{ }^{*} P \leq 0.01,{ }^{* *} P \leq 0.001$, $* * * P \leq 0.0001$.

levels were reversed (Fig. 6A). The analysis via Phospholipid Kits showed the consistent results (Fig. 6B). These indicated that SFNinhibited mitophagy contributed the decrease of FA. Reversely, the decrease might inhibit mitophagy. After knocking down of atubulin, mitophagy markers BNIP3 and NIX levels were downregulated (Fig. 6C, D). Meanwhile, LC3 II/I levels were upregulated (Fig. 6E). These indicated that SFN inhibited the binding of mitophagosome to lysosome by decreasing a-tubulin. Coimmunoprecipitation showed that the interaction between a-tubulin and NIX was downregulated in both the mitochondria and cytoplasm (Fig. 6F, G). Confocal microscopy observation showed that SFN inhibited the colocalization of a-tubulin to NIX (Fig. $6 \mathrm{H}$ ). These results indicated that SFN inhibited mitophagy regulating $F A$ level via a microtubule-mediated way. Flow cytometry showed that cell apoptosis rates were decreased after co-treated with CCCP and the apoptosis rates were increased after co-treated with Baf-A1 (Fig. 6l, J). Taken together, these data demonstrated that SFN inhibited microtubulemediated FA synthesis and mitophagy leading to apoptosis (Fig. 6K). 
A
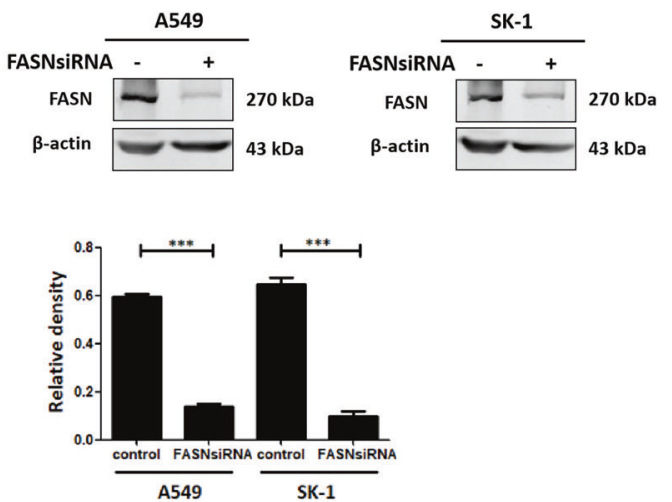

C
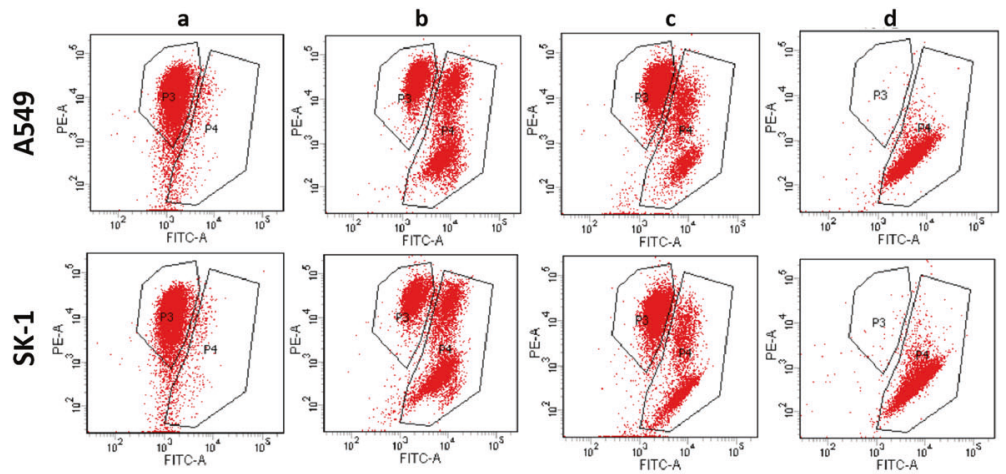

D

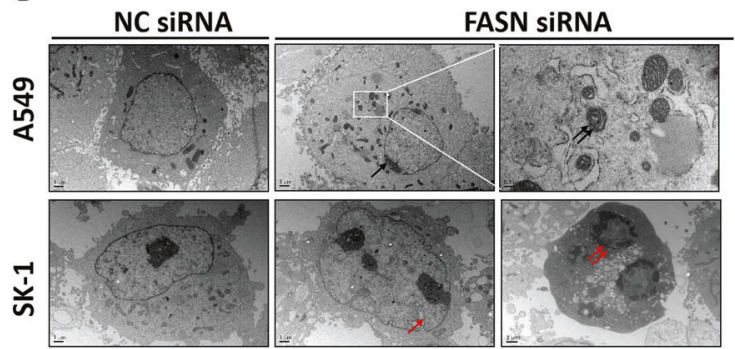

$\mathbf{F}$

G
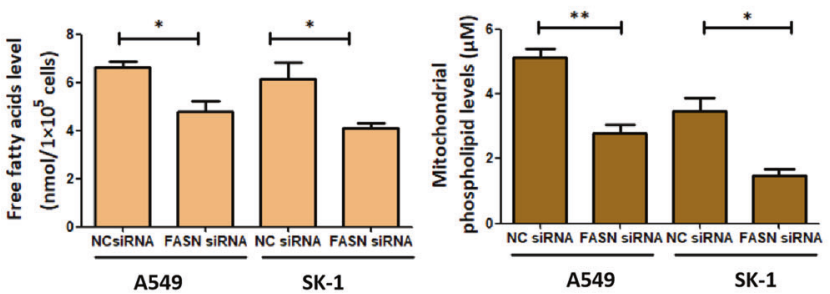

H

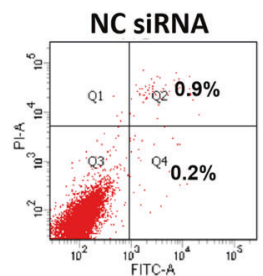

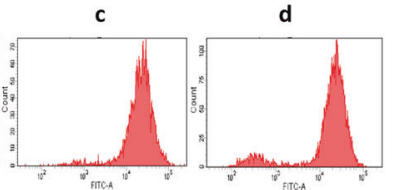
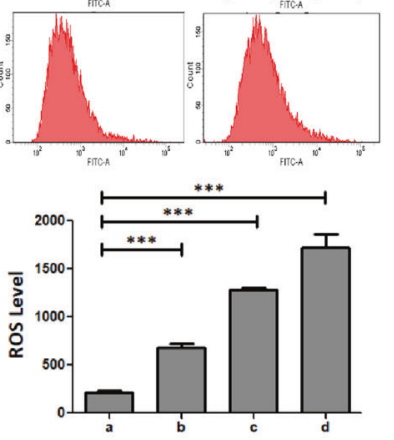

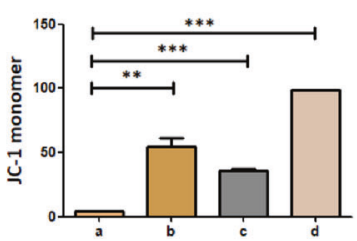

SK-1
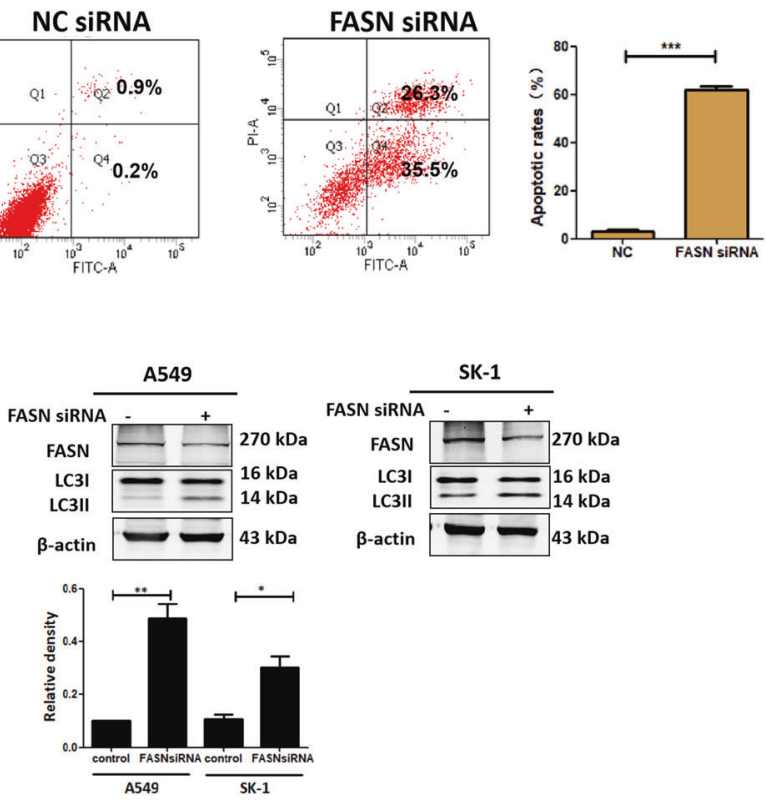

mechanisms that SFN triggered apoptosis in the subcellular level. HPLC-MS/MS analysis showed that FASN, ACACA, a-tubulin, and $\beta$-tubulin were the target molecules, which SFN initiated anticancer signaling to downregulate. Previous studies showed that SFN might bind to $\alpha$-tubulin, $\beta$-tubulin to degrade $\alpha$-tubulin, and $\beta$-tubulin causing apoptosis [26]. a-tubulin is the main component 
Fig. 4 SFN-triggered downregulation of FASN level contributed to apoptosis. A FASN accumulation was detected by western blotting after knockdown of FASN via siRNA, both in A549 and SK-1 cells. B Flow cytometry was used to detect ROS levels after knocking down FASN mRNA. (a: NC siRNA, b: FASN siRNA, c: $20 \mu \mathrm{M}$ SFN, d: FASN siRNA plus $20 \mu \mathrm{M}$ SFN). C Flow cytometry was used to detect JC-1 levels after knocking down FASN mRNA in cells (a: NC siRNA, b: FASN siRNA, c: $20 \mu \mathrm{M}$ SFN, d: FASN siRNA plus $20 \mu \mathrm{M}$ SFN). D After knocking down FASN mRNA in cells, cells were observed via TEM. Red arrows indicate membrane disruption, black arrows indicate chromatin edge aggregation, double black arrows indicate swollen mitochondria and double red arrows indicated nuclear fragments. Scale bar: $1 \mu \mathrm{m}$. E Cells were transfected with NC siRNA or FASN siRNA, flow cytometry was used to detect apoptosis rates. F Determination of total free FA content after FASN was knocked down. G Mitochondrial phospholipid content was detected by kit after FASN was knocked down. H After knockdown of FASN level, western blotting was used to analyze LC3 II/I accumulation. Data were shown as means \pm SEM $(n \geq 3) .{ }^{*} P \leq 0.01 ;{ }^{* *} P \leq 0.001 ;{ }^{* * *} P \leq 0.0001$.

of microtubules and might bind tightly to $\beta$-tubulin to form microtubule dimers [27]. Microtubule acts as the cytoskeleton to support a variety of proteins for transport and scaffolding $[28,29]$. Here we further uncovered that SFN inhibited the formation of dimers consisting of $a$-tubulin and $\beta$-tubulin, resulting in microtubule depolymerization. These results indicated that SFNtriggered microtubule disruption played a role in FA synthesis and metabolism.

Interestingly, SFN treatment caused a decrease in total free FA and mitochondrial phospholipids. Also, the levels of a few lipid enzymes involved in FA synthesis were also decreased upon SFN treatment in cultured cancer cells. FA is critical for energy metabolism and is the fundamental component of all cell membrane [30]. FASN is a key enzyme involved in the synthesis of FA essential for the de novo synthesis of FA in tumor cells $[10,31]$. Therefore, it is helpful to establish a new anti-cancer therapy via targeting FA synthesis and metabolism signaling. TVB2640 is a clinically effective FASN inhibitor, which works greatly in the treatment of patients with NSCLC [10]. However, most FASN inhibitors are not used for clinical study due to poor stability and insufficient bioavailability such as cerulenin and orlistat. It was expected that SFN would have an excellent oral bioavailability, because SFN exerted a synergistically anti-cancer effect in combination with paclitaxel reducing the dosage of both agents $[32,33]$. Moreover, NSCLC patients with higher level of FASN have lower survival. By immunohistochemical staining in microarray tissues, we found that FASN was highly expressed in tumors and was associated with malignant grades of tumors. As the most critical transcription factor regulating lipid synthesis, SREBP1 is essential for the de novo synthesis of FA [34]. It was reported that $A C L Y, A C A C A$, and FASN levels were positively correlated to SREPB1 $[35,36]$. Studies showed that SFN inhibited lipid synthesis by inhibiting key enzymes such as FASN and ACACA in prostate cancer [13]. Both ACACA and ACLY are the rate-limiting enzymes in de novo synthesis of FA and here their levels were decreased in response to SFN. Our results showed that SFN degraded SREBP1 and a few key enzymes in lipid synthesis through the $26 \mathrm{~S}$ proteasome pathway. Further, knockdown of FASN caused damages in cell membrane structure and mitochondrial morphology. Mitochondria acted as the sites for ROS production and mitochondrial dysfunction induced the production of ROS. Excessive ROS might further damage the mitochondria and the accumulation of damaged mitochondria might cause apoptosis [37]. Therefore, SFN might interrupt microtubule dynamics and interfere with lipid metabolism, leading to cell death. These helped us understand SFN signaling and function in the energy metabolism.

Studies showed that the changes in membrane lipids might disrupt membrane fluidity and interfere with the normal fusion of two membrane-bound organelles, such as autophagosome and lysosome [38]. It was reported that FASN inhibitors increased the accumulation of LC3 II in a couple of cancers [39, 40]. Here, SFN inhibited FASN production, resulting in decreased levels of total free FA and mitochondrial total phospholipids and mitochondrial damage. Mitophagy served as a negative feedback maintaining cell homeostasis. As a result, SFN prevented cells from repairing damaged mitochondria by inhibiting mitophagy leading to apoptosis.

The autophagosome membrane derived from the mitochondria and the impaired mitochondrial membrane significantly prevented the formation of autophagic spots, which might be caused by a decrease in mitochondrial phospholipids. The phospholipid in the mitochondrial membrane was mainly cardiolipin, located in the mitochondrial inner membrane, and it was transferred to the outer membrane when the mitochondria was damaged, triggering mitophagy [41, 42]. Our results showed that the total free FA and mitochondrial phospholipids were increased after combined with CCCP. This suggested that SFN decreased mitochondrial phospholipid levels, thereby disrupting the mitochondrial membrane. Destruction of integrity of the mitochondrial membrane might be one of the reasons to inhibit the formation of autophagosomes and mitophagy [43]. On the contrary, decreased FA levels might inhibit mitophagy leading to apoptosis.

In NSCLC, we reported that SFN metabolites inhibited microtubule-mediated autophagy leading to apoptosis [44]. Here we found more profound mechanisms in the subcellular level. SFN mainly inhibited microtubule-mediated FA production and microtubule-regulated mitophagy affecting energy metabolism. Mitochondria acted as dynamic organelles that constantly modulated their morphology, function, and quantity to accommodate the metabolic needs of cells. Mitophagy might effectively remove damaged mitochondria by PINK/Parkin, BNIP3/NIX, and FOUNDC1 pathways [45]. The accumulation of dysfunctional mitochondria was associated with many human diseases including cancer [46]. It was reported that BNIP3L/NIX regulated the removal of the mitochondria. This mitophagy receptor bound to the mitochondria and phagocytic membrane protein LC3 II/I, and promoted mitochondrial encapsulation in autophagosomes, then it was delivered to lysosomes for degradation [46]. Both NIX and BNIP3 localized to the outer mitochondrial membrane by way of a C-terminal transmembrane domain [47]. Also, both of them directly interacted with the autophagosome membrane protein LC3 [48]. Here, we found that SFN inhibited mitophagy via downregulating BNIP3 and NIX. Further, our study showed that SFN inhibited the interaction between NIX and LC3. These results demonstrated that SFN inhibited the formation of mitophagosomes. The interaction between a-tubulin and NIX was determined after treated with SFN. After knockdown of a-tubulin, the levels of BNIP3 and NIX were downregulated, indicating that SFN induced microtubule disruption resulting in inhibition of mitophagy.

Although autophagy was discovered for more than 50 years, the relationship between mitophagy and cell death remained elusive [49]. It was reported that autophagy protected cells by maintaining energy homeostasis and nutrient requirements under starvation conditions. In tumor cells, autophagy might contribute to the undesired survival of tumor cells [50,51]. By cotreatment with mitophagy inducer CCCP, we demonstrated that SFN decreased FA levels inhibiting mitophagy, leading to apoptosis in NSCLC cells. These gave us some new hints that SFN might disturb microtubule-mediated synthesis and mitophagy. Meanwhile, SFN might interfere with the cross-talks between FA metabolism and mitophagy. 
A
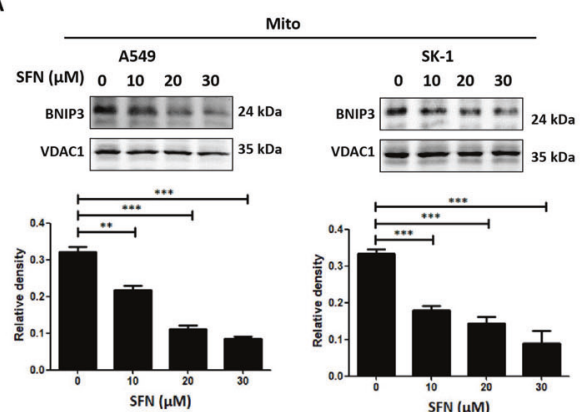

C

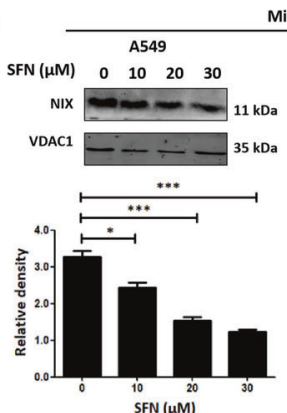

Mito

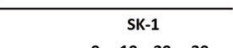

$\begin{array}{llll}0 & 10 & 20 & 30\end{array}$

$\mathrm{NIX}=-11 \mathrm{kDa}$

VDAC1 $-.-1-35 \mathrm{kDa}$
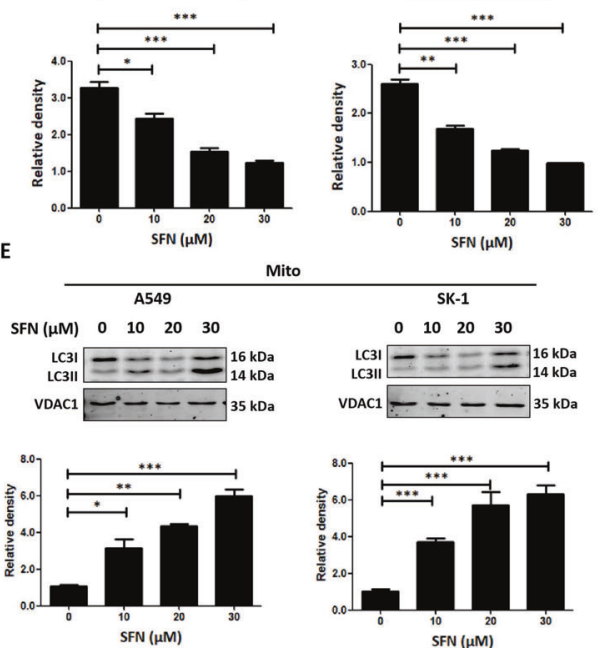
\begin{tabular}{|cccc} 
& SK-1 \\
0 & 10 & 20 & 30
\end{tabular}

$\mathrm{LC}_{\mathrm{L} 31}= \pm=\equiv{ }_{14 \mathrm{kDa}}^{16}$ vDAC1 $-\div-\div 35 \mathrm{kDa}$

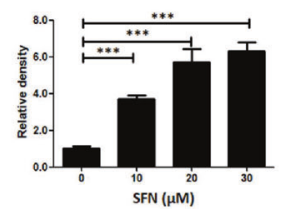

G

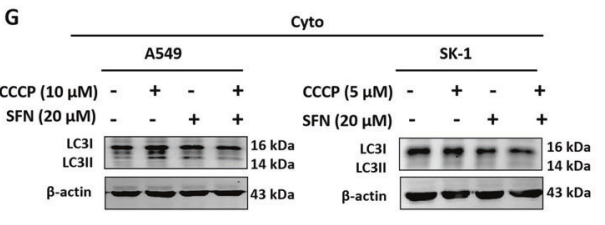

I

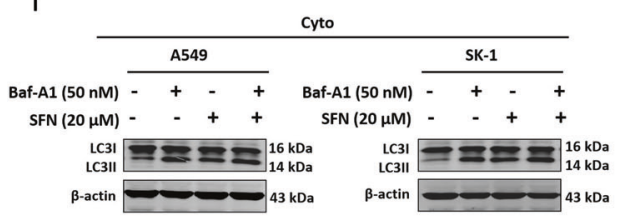

K

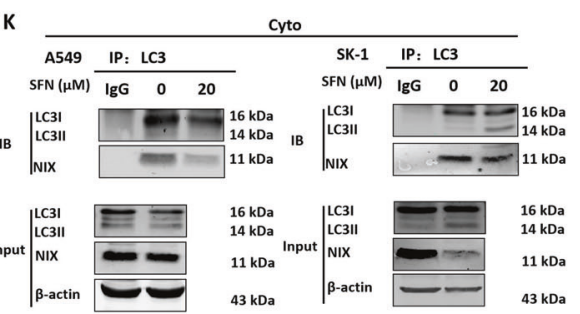

Taken together, we uncovered a brand new mechanism that SFN induced apoptosis via inhibiting microtubule-mediated FA synthesis and mitophagy. These results might help us to establish new anti-cancer therapies via targeting lipid signaling and mitophagy-associated molecules.
B
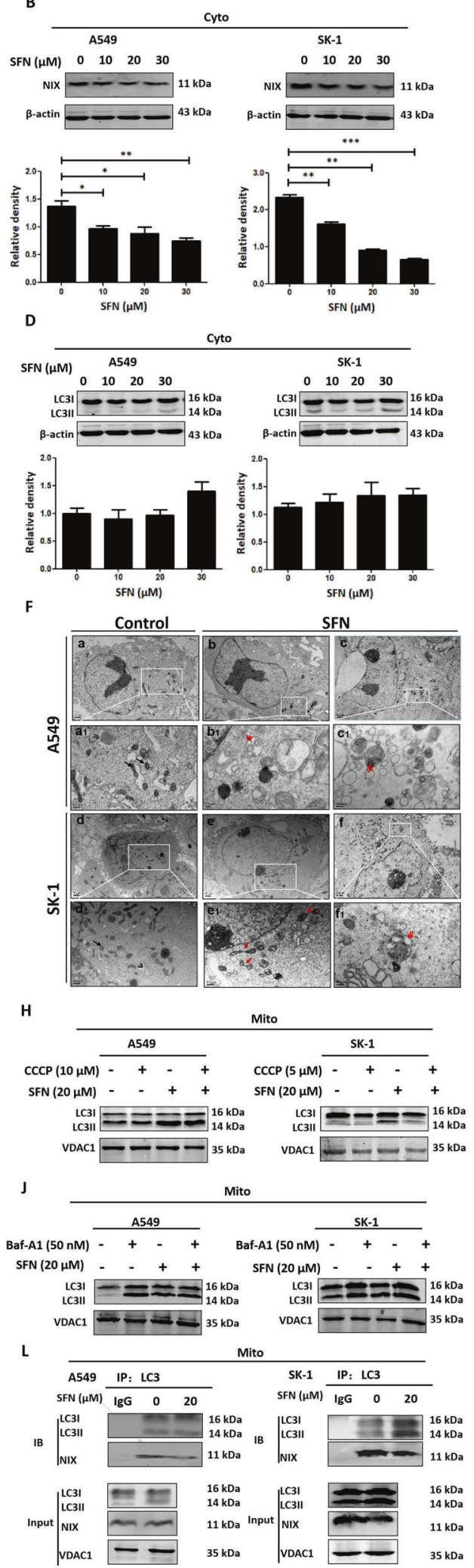

MATERIALS AND METHODS

Antibodies and reagents

Anti-a-tubulin (YM3035) and anti- $\beta$-tubulin (YM3139) were purchased from ImmunoWay Biotechnology (TX, USA); anti-FASN (SC-48357), anti-NIX (SC166332), and anti-SREBP1 (SC-13551) were purchased from Santa Cruz Biotech (TX, USA); anti-BNIP3 (D121876) and anti-ACLY (D221957) were 
Fig. 5 SFN inhibited microtubule-mediated mitophagy and the fusion of mitophagosomes to lysosomes. After SFN was used at a designated concentration for $24 \mathrm{~h}$, the mitochondrial isolation kit was used to separate the cytoplasm and mitochondrial protein lysates. Western blotting was used to analyze BNIP3 level in the mitochondria. B, C After SFN was used at a designated concentration for $24 \mathrm{~h}$, the mitochondrial isolation kit was used to separate the cytoplasmic and mitochondrial proteins. Western blotting was used to analyze NIX level in the cytoplasm (B) and mitochondria (C). D, E After SFN was used at a designated concentration for $24 \mathrm{~h}$, the mitochondrial isolation kit was used to separate the cytoplasmic and mitochondrial proteins. Western blotting was used to analyze LC3 II/I accumulation in the cytoplasm (D) and mitochondria (E). F Cells were treated with/without $20 \mu \mathrm{M}$ SFN for $24 \mathrm{~h}$. The images via TEM showed the mitophagosomes; double red arrows marked mitophagosomes; black arrows marked normal mitochondria; red arrows marked swollen mitochondria. Scale bar: $1 \mu \mathrm{m}$ (a-f), $0.5 \mu \mathrm{m}\left(a_{1}-f_{1}\right)$. G, H Both A549 and SK-1 cells were pretreated with CCCP $(10$ or $5 \mu \mathrm{M})$ for $2 \mathrm{~h}$, then treated with SFN $(20 \mu \mathrm{M})$ for $24 \mathrm{~h}$, the mitochondrial isolation kit was used to separate the cytoplasmic and mitochondrial proteins. The cytoplasm (G) or mitochondria (H) accumulation of LC3 II/I was analyzed by western blotting. I, J Both A549 and SK-1 cells were pretreated with Baf-A1 (50 nM) for $2 \mathrm{~h}$, then treated with SFN $(20 \mu \mathrm{M})$ for $24 \mathrm{~h}$, the mitochondrial isolation kit was used to separate the cytoplasm and mitochondrial proteins. The cytoplasm (I) or mitochondria (J) accumulation of LC3 II/I was analyzed by western blotting. K, L After SFN was used at a designated concentration for $24 \mathrm{~h}$, the mitochondrial isolation kit was used to separate the cytoplasmic and mitochondrial proteins. The binding of LC3 II/ I to NIX was detected in both cytoplasm $(\mathbf{K})$ and mitochondria (L) by coimmunoprecipitation. Data were shown as means \pm SEM $(n \geq 3)$. ${ }^{*} P \leq$ $0.01 ;{ }^{* *} P \leq 0.001 ;{ }^{* * *} P \leq 0.0001$.

purchased from Sangon Biotech(Shanghai, China); anti-ACACA (21923-1-AP) and anti- $\beta$-actin (66009-1-Ig) were purchased from Proteintech Group (IL, USA); anti-LC3 II/I (D3U4C) and anti-caspase-3 (D3R6Y) were purchased from Cell Signaling Technology (MA, USA); anti-VDAC1 (AB14734) was purchased from Abcam (MA, USA); D, L-Sulforaphane (574215) was purchased from Sigma-Aldrich (MO, USA); CCCP (C2759) was purchased from Sigma-Aldrich (MO, USA); Baf-A1 (S1413) and PS-341 (S1013) were purchased from Selleck (Shanghai, China); Cell Mitochondria Isolation Kit (C3601), ROS Assay Kit (S0033), and Mitochondrial Membrane Potential Assay Kit (C2006) were purchased from Beyotime Biotechnology (Shanghai, China).

\section{Cell line}

Human lung adenocarcinoma cell line A549 and human lung squamous carcinoma cell line SK-1 were purchased and justified from the Cell Resource Center, Peking Union Medical College (Beijing, China).

\section{Cell culture and transfection}

Cells were incubated in Dulbecco's modified Eagle medium/F-12 culture medium with $10 \%$ fetal bovine serum (Gibco, 16,000-044) and $100 \mathrm{U} / \mathrm{ml}$ penicillin and streptomycin. All cells were cultured in a humidified incubator containing $5 \% \mathrm{CO}_{2}$ at $37^{\circ} \mathrm{C}$

For knockdown of FASN mRNA, negative control siRNA (5'- UUCUCCGAACGUGUCACGUTT-3'), FASN siRNA (5'-CAGAGUCGGAGAACUUGCAGGAGUU-3'), a-tubulin siRNA (5'-AAAGATGTCAATGCTGCCATT-3'), and $\beta$-tubulin siRNA (5'CCCAGCGGCAACTACGTGGG-3') were designed [1, 16, 24], SREBP1 siRNA was purchased from Santa Cruz Biotech (sc-36558). Cells were plated in six-well plates at a density of $1 \times 10^{6} /$ well and cultured for $24 \mathrm{~h}$. Then the cells were transfected with the FASN and a-tubulin siRNA, respectively ( $30 \mathrm{pmol} /$ well) by Lipofectamine ${ }^{\text {TM }}$ RNAiMAX (Invitrogen, 13778075) when cells reach $\sim 80 \%$ confluency.

\section{Mitochondrial protein extraction}

Cells $\left(2 \times 10^{7}\right)$ treated with or without $20 \mu \mathrm{M} \mathrm{SFN}$ for $24 \mathrm{~h}$ were collected and washed once in ice-cold phosphate-buffered saline (PBS). Mitochondrial proteins were isolated from the cell by the Cell Mitochondria Isolation Kit according to the manufacturer's instructions. Mitochondrial protein concentrations were determined by the BCA Protein Assay Kit (Invitrogen, 23227) and cytoplasm protein concentrations were determined by the Bradford assay (Applygen, P1510).

\section{Western blotting}

Cells were lysed by RIPA buffer (Thermo Fisher Scientific, 89900) supplemented with protease inhibitors cocktail. Whole-cell lysates were quantified by the BCA Protein Assay Kit. Equal quantity of protein molecules $(30 \mu \mathrm{g})$ from each sample were separated by $8 \%$ or $12 \%$ SDSpolyacrylamide gel electrophoresis. The procedures were carried out according to the previous experimental method [2].

\section{Coimmunoprecipitation}

After washed with ice-cold PBS, the cells were lysed on ice via Nondenaturing Lysis Buffer supplemented with protease inhibitors cocktail. The monoclonal anti-a-tubulin or anti- $\beta$-tubulin, or anti-FASN or anti$\mathrm{NIX}$, or anti-LC3 II/I was added into the protein lysates and the mixture was incubated overnight at $4{ }^{\circ} \mathrm{C}$. The complexes were pulled down with protein
A/G agarose for $3 \mathrm{~h}$ and the proteins were isolated by centrifuging and boiling for $5 \mathrm{~min}$. Western blotting was used to recognize the conjugated proteins.

\section{Immunofluorescence staining}

Cells were seeded in $35 \mathrm{~mm}$ cover glass-bottom dishes at a density of $1 \times$ $10^{5}$ cells/dish and incubated for $24 \mathrm{~h}$, then treated with $20 \mu \mathrm{M} \mathrm{SFN}$ for $24 \mathrm{~h}$. These cells were fixed with $1 \%$ paraformaldehyde for $15 \mathrm{~min}$. Then the cells were washed for three times with PBST (PBS plus Tween-20) and permeabilized with ice-cold methanol for $10 \mathrm{~min}$ at room temperature. After blocking with PBS containing $1 \%$ bovine serum albumin and $0.1 \%$ Triton X-100 for $1 \mathrm{~h}$, the cells were incubated overnight at $4^{\circ} \mathrm{C}$ with the corresponding primary antibodies. Then the cells were washed for three times with PBST and incubated with the fluorescence-labeled secondary antibody for $1 \mathrm{~h}$ at room temperature. After washing with PBST for three times, the cells were stained with 4',6-diamidino-2-phenylindole (Zsgb-bio, ZLI-9557). Fluorescence images were collected under a laser scanning confocal microscope (Olympus FV1000; Olympus Corp., Tokyo, Japan).

\section{Microtubule polymerization assay}

The collected cells were washed twice with PBS, then lysed at $37^{\circ} \mathrm{C}$ for 30 min with $400 \mu \mathrm{L}$ lysis buffer $(20 \mathrm{mM}$ Tris- $\mathrm{HCl}$ pH $6.8,1 \mathrm{mM} \mathrm{MgCl} 2,2 \mathrm{mM}$ EGTA, 1\% NP-40) with Protease Inhibitor Cocktail (Roche, 04693132001). The cell lysates were centrifuged at 12,500 r.p.m. for $15 \mathrm{~min}$ at $25^{\circ} \mathrm{C}$. The supernatant containing soluble a-tubulin was collected, whereas the pellet containing assembled a-tubulin was suspended in $40 \mu \mathrm{L}$ of pellet lysis buffer (20 mM Tris-HCl pH 6.8, $1 \mathrm{mM} \mathrm{MgCl} 2,2 \mathrm{mM}$ EGTA, 2\% SDS) after washing with PBS. Then the precipitate was heated at $95^{\circ} \mathrm{C}$ for 30 min until the pellet was solved. These a-tubulin molecules in two fractions (soluble and insoluble) were separated by western blotting.

\section{Transmission electron microscopy}

Cells were treated with $20 \mu \mathrm{M}$ SFN for $24 \mathrm{~h}$. After being collected and washed with PBS for two times, the sample was fixed with $3 \%$ glutaraldehyde at $4{ }^{\circ} \mathrm{C}$ for $2 \mathrm{~h}$. After washing with PBS for three times, the sample was fixed in $1 \%$ osmium tetroxide for $1 \mathrm{~h}$. Samples were dehydrated through a series of concentrations of ethanol, and infiltrated and embedded in a 1:1 mixture of acetone and Epon-812 resin for $30 \mathrm{~min}$. The samples were infiltrated in Epon-812 for $2 \mathrm{~h}$, cut into ultrathin sections with a knife, and positioned on 200-mesh copper grids. Sections were stained with Uranium acetate for $30 \mathrm{~min}$ and then stained with Lead nitrate for $20 \mathrm{~min}$. The sections were then observed and photographed with a TEM (JEM-2100Plus, JEOL, Ltd, Tokyo, Japan).

\section{HPLC-MS/MS analysis}

HPLC-MS/MS was used to analyze the production in A549 cells after treated with or without SFN. Cells were treated with SFN for $24 \mathrm{~h}$, then the cell lysates were collected and quantified. Equal quantity of protein molecules were analyzed by HPLC-MS/MS. Chromatographic separation and analysis were performed by a Orbitrap Fusion Lumos mass spectrometer (Thermo Fisher Scientific, USA) and an EASY-nLC 1000 liquid chromatography system (Thermo Fisher Scientific, USA) equipped with an electrospray ionization source. Separation was carried out by a C18 


\section{A}

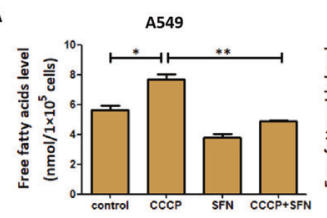

C

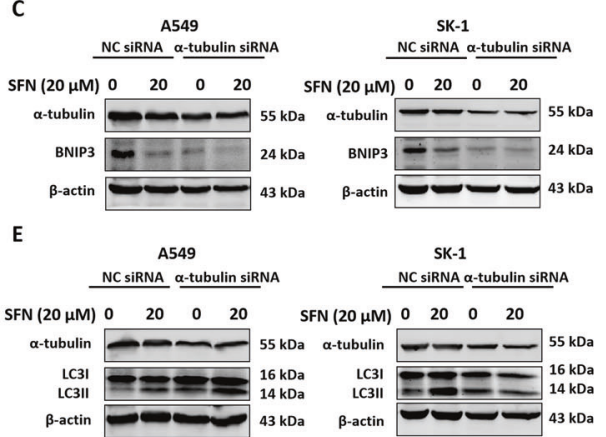

G

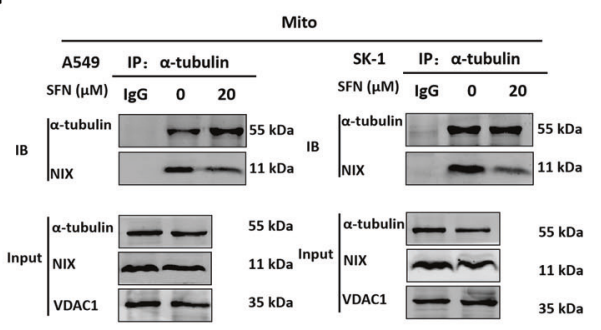

I
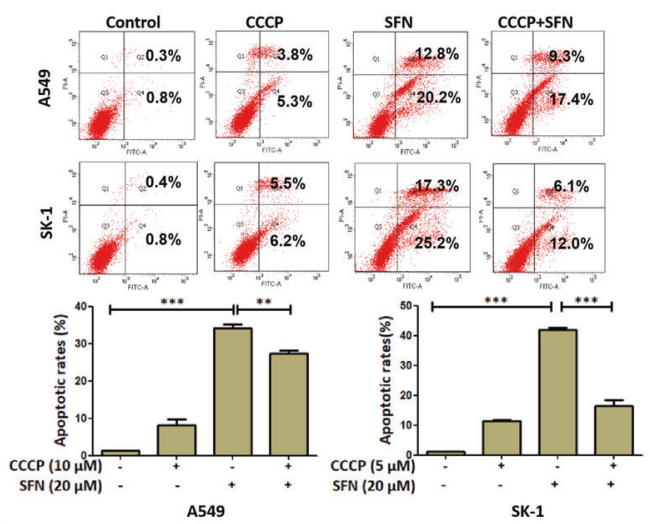

K

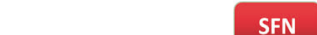

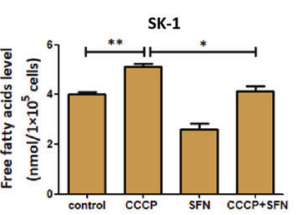

D
B

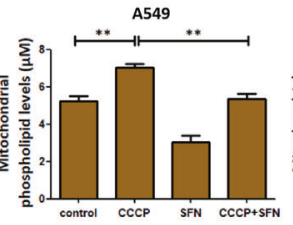
A549
NC siRNA $\alpha$-tubulin siRNA

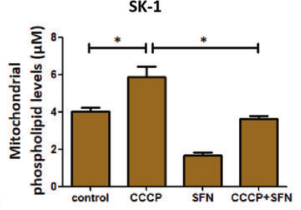

$\operatorname{SFN}(20 \mu \mathrm{M}) 0 \quad 20 \quad 0 \quad 20 \quad \operatorname{SFN}(20 \mu \mathrm{M}) \begin{array}{lllll}0 & 20 & 0 & 20\end{array}$ $\alpha$-tubulin $-\longrightarrow 55 \mathrm{kDa}$ a-tubulin $\longrightarrow-\longrightarrow 55 \mathrm{kDa}$

NIX -

$\beta$-actin $20-33$ kDa $\quad \beta$-actin

F

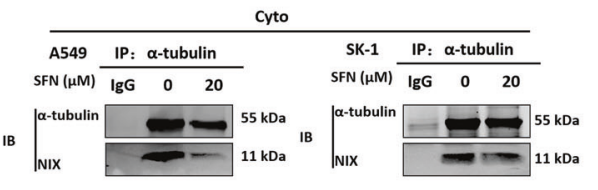

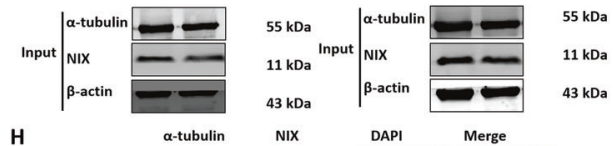

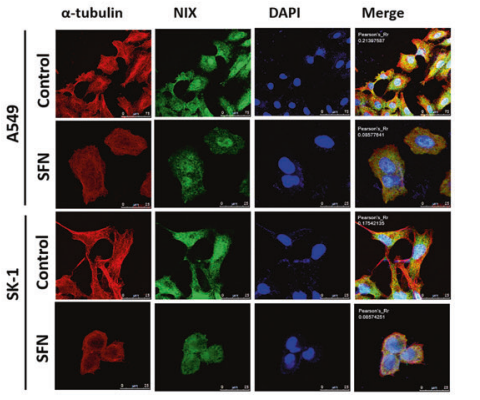

$\begin{array}{llll}J & \text { Control Baf-A1 } & \text { SFN } & \text { Baf-A1+SFN }\end{array}$

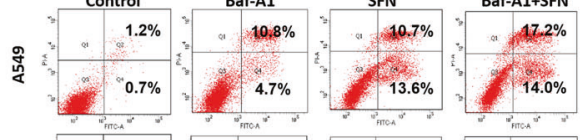

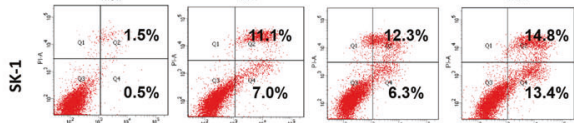
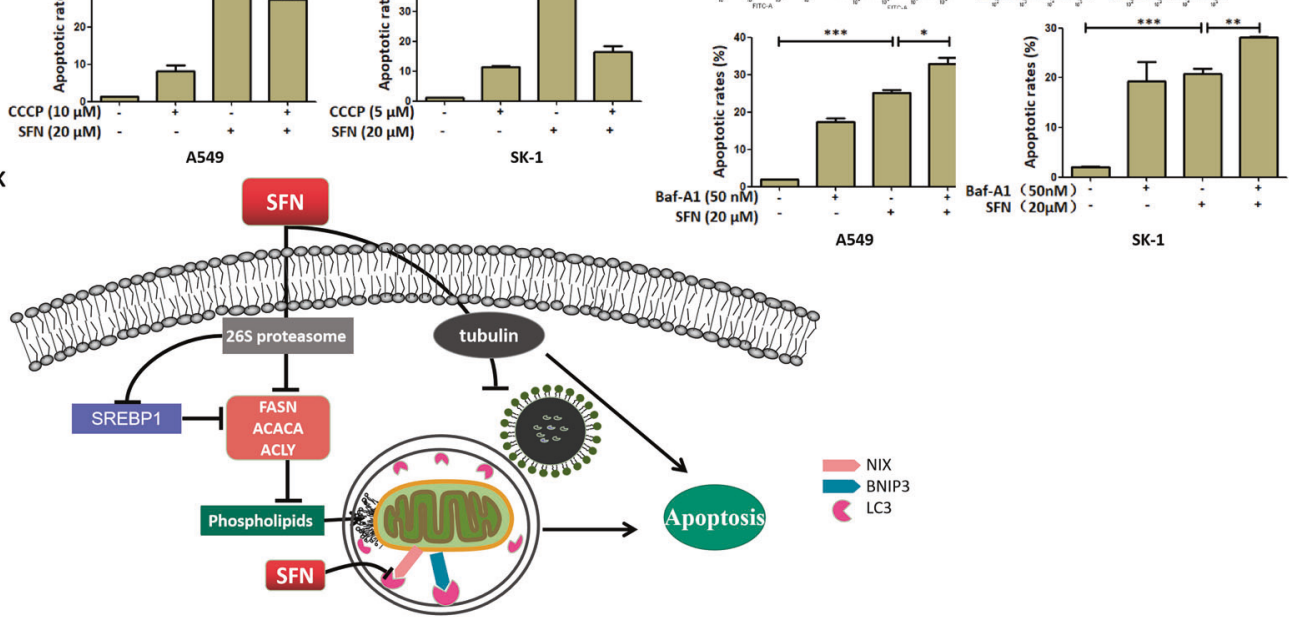

$(1.9 \mu \mathrm{m}, 100 \AA)$ capillary column with a mobile phase of $0.1 \% \mathrm{HCOOH} / \mathrm{H}_{2} \mathrm{O}$ and $0.1 \% \mathrm{HCOOH} / \mathrm{ACN}$. Each sample was injected twice to perform detection in positive ionization modes. After the collected data were identified, proteins were analyzed by the Uniprot net for cell localization and function.
Tissue microarray immunohistochemistry

Human lung cancer tissue microarrays with 150 patient samples and different Gleason patterns were established by Shanghai Biochip (Shanghai, China). The immunohistochemistry stain was done with human-specific anti-FASN combined with the UltraSensitive ${ }^{\mathrm{TM}} \mathrm{S}$-P detection 
Fig. 6 SFN induced apoptosis by inhibiting microtubule-mediated FA synthesis and mitophagy. A, B Both A549 and SK-1 cells were pretreated with CCCP $(10$ or $5 \mu \mathrm{M})$ for $2 \mathrm{~h}$, then treated with SFN $(20 \mu \mathrm{M})$ for $24 \mathrm{~h}$, determination of total free FA (A) and mitochondrial phospholipid content (B) was determined. C-E Both A549 and SK-1 cells were transfected with NC siRNA or $\alpha$-tubulin siRNA, and treated with SFN $(20 \mu \mathrm{M})$ for $24 \mathrm{~h}$, the accumulation of BNIP3 (C) or NIX (D), or LC3 I /II (E) was determined by western blotting. F, G Both A549 and SK-1 cells were treated with SFN $(20 \mu \mathrm{M})$ for $24 \mathrm{~h}$, the mitochondrial isolation kit was used to separate the cytoplasmic and mitochondrial proteins. Then binding of $\alpha$-tubulin to NIX was detected both in the cytoplasm (F) and mitochondria (G) by coimmunoprecipitation. $\mathbf{H}$ Immunofluorescence and confocal microscopy were employed to observe the colocalization of $\alpha$-tubulin to NIX. Scale bar: A549: $75 \mu \mathrm{m}, \mathrm{SK}-1$ : control, $25 \mu \mathrm{m}$, SK-1: SFN, $25 \mu \mathrm{m}$. I, J Both A549 and SK-1 cells were pretreated with CCCP $(10$ or $5 \mu \mathrm{M})$ (I) or Baf-A1 (50 nM) (J) for $2 \mathrm{~h}$, then treated with SFN (20 $\mu \mathrm{M})$ for $24 \mathrm{~h}$, then the cells were collected and the percentage of apoptosis rates were analyzed by flow cytometer via Annexin V-FITC/PI Apoptosis Detection Kit. K A possible schematic of the involved signal pathways that SFN induced apoptosis via inhibiting microtubule-mediated FA synthesis and mitophagy in NSCLC cells. Data were shown as means \pm SEM $(n \geq 3)$. ${ }^{*} P \leq 0.01 ;{ }^{* *} P \leq 0.001 ;{ }^{* * *} P \leq 0.0001$.

kit (Maixin, KIT-9710). The protocol was derived from the published paper [16].

\section{Apoptosis assay}

Cell apoptosis was measured via Annexin V-FITC apoptosis assay kit (GenStar, C203-01). Cells were collected and washed twice with ice-cold PBS, then these cells were resuspended in $250 \mu \mathrm{L} 4 \times$ binding buffer. Then $5 \mu \mathrm{L}$ Annexin V-FITC and $10 \mu \mathrm{L}$ Propidium lodide were added to $100 \mu \mathrm{L}$ cell suspension, and the reaction was incubated for $5 \mathrm{~min}$ at room temperature in the dark. Apoptotic cells were detected via a flow cytometer.

\section{Free FA quantification assay}

Levels of total free FAs were determined by Free Fatty Acid Quantitation kit (Sigma, MAK044). Cells $\left(1 \times 10^{6}\right)$ were collected and resuspended in $200 \mu \mathrm{L}$ of $1 \%(\mathrm{w} / \mathrm{v})$ Triton X-100 in chloroform solution. The homogenates were centrifuged at 13,000 r.p.m. for $10 \mathrm{~min}$ and we collected the organic phases to accept vacuum dry for $30 \mathrm{~min}$, to remove trace chloroform. The dried lipids were dissolved in $200 \mathrm{~L}$ of Fatty Acid Assay Buffer for detection.

\section{Mitochondrial phospholipids quantification assay}

Cells $\left(1 \times 10^{6}\right)$ were collected to Isolate mitochondria with the kit. These mitochondria were lysed with $1 \% \mathrm{NP}-40$ for $30 \mathrm{~min}$. Cell mitochondrial phospholipids were measured via Phospholipid Assay Kit (Sigma, MAK122).

\section{Bioinformatics analysis}

GEPIA (Gene Expression Profiling Interactive Analysis) is a public database newly developed by the Chinese for cancer and normal gene expression profiling, from 9736 tumors and 8587 normal samples from The Cancer Genome Atlas and Genotype-Tissue Expression projects. We searched the GEPIA Database to determine the genetic correlation in lung cancer. The version 10.5 of STRING database was used to find the interaction among NIX and lipid metabolism-related proteins [52].

\section{Statistical analysis}

All data were expressed as mean \pm SEM from three independent experiments. Paired data were evaluated by Student's $t$-test. Two-way analysis of variance was used to determine statistical significance. $P \leq 0.05$ was considered statistically significant. All statistical analyses were performed by SPSS version 19.0.

\section{DATA AVAILABILITY}

All data generated or analyzed during this study are included in this published article.

\section{REFERENCES}

1. Hu Y, Zhou Y, Yang G, Wang Y, Zheng Z, Li J, et al. Sulforaphane-N-acetyl-cysteine inhibited autophagy leading to apoptosis via Hsp70-mediated microtubule disruption. Cancer Lett. 2018;431:85-95.

2. Tian H, Zhou Y, Yang G, Geng Y, Wu S, Hu Y, et al. Sulforaphane-cysteine suppresses invasion via downregulation of galectin-1 in human prostate cancer DU145 and PC3 cells. Oncol Rep. 2016;36:1361-8.

3. Geng $Y$, Zhou $Y$, Wu S, Hu Y, Lin K, Wang Y, et al. Sulforaphane induced apoptosis via promotion of mitochondrial fusion and ERK1/2-mediated $26 \mathrm{~S}$ proteasome degradation of novel pro-survival bim and upregulation of Bax in human nonsmall cell lung cancer cells. J Cancer. 2017;8:2456-70.
4. Huang C, Freter C. Lipid metabolism, apoptosis and cancer therapy. Int J Mol Sci. 2015;16:924-49.

5. Rohrig F, Schulze A. The multifaceted roles of fatty acid synthesis in cancer. Nat Rev Cancer. 2016;16:732-49.

6. Singh A, Ruiz C, Bhalla K, Haley J, Li Q, Acquaah-Mensah G, et al. De novo lipogenesis represents a therapeutic target in mutant Kras non-small cell lung cancer. FASEB J. 2018;32:7018-27.

7. Ali A, Levantini E, Teo JT, Goggi J, Clohessy JG, Wu CS, et al. Fatty acid synthase mediates EGFR palmitoylation in EGFR mutated non-small cell lung cancer. EMBO Mol Med. 2018;10:e8313.

8. Ventura R, Mordec K, Waszczuk J, Wang Z, Lai J, Fridlib M, et al. Inhibition of de novo palmitate synthesis by fatty acid synthase induces apoptosis in tumor cells by remodeling cell membranes, inhibiting signaling pathways, and reprogramming gene expression. EBioMedicine. 2015;2:808-24.

9. Menendez JA, Vazquez-Martin A, Ortega FJ, Fernandez-Real JM. Fatty acid synthase: association with insulin resistance, type 2 diabetes, and cancer. Clin Chem. 2009;55:425-38.

10. Jones SF, Infante JR. Molecular pathways: fatty acid synthase. Clin Cancer Res. 2015;21:5434-8.

11. Wei $X$, Song $H$, Yin L, Rizzo MG, Sidhu R, Covey DF, et al. Fatty acid synthesis configures the plasma membrane for inflammation in diabetes. Nature. 2016;539:294-8.

12. Svensson RU, Parker SJ, Eichner LJ, Kolar MJ, Wallace M, Brun SN, et al. Inhibition of acetyl-CoA carboxylase suppresses fatty acid synthesis and tumor growth of non-small-cell lung cancer in preclinical models. Nat Med. 2016;22:1108-19.

13. Singh KB, Kim SH, Hahm ER, Pore SK, Jacobs BL, Singh SV. Prostate cancer chemoprevention by sulforaphane in a preclinical mouse model is associated with inhibition of fatty acid metabolism. Carcinogenesis. 2018;39:826-37.

14. Currie E, Schulze A, Zechner R, Walther TC, Farese RV Jr. Cellular fatty acid metabolism and cancer. Cell Metab. 2013;18:153-61.

15. Su $X$, Jiang $X$, Meng L, Dong $X$, Shen $Y$, Xin Y. Anticancer activity of sulforaphane: the epigenetic mechanisms and the Nrf2 signaling pathway. Oxid Med Cell Longev. 2018;2018:5438179.

16. Wang Y, Zhou Y, Zheng Z, Li J, Yan Y, Wu W. Sulforaphane metabolites reduce resistance to paclitaxel via microtubule disruption. Cell Death Dis. 2018;9:1134.

17. Flemming A. Cancer: Microtubule-straightening compound widens the therapeutic window. Nat Rev Drug Discov. 2016;16:17.

18. Schaaf MB, Keulers TG, Vooijs MA, Rouschop KM. LC3/GABARAP family proteins: autophagy-(un)related functions. FASEB J. 2016;30:3961-78.

19. Kerr JS, Adriaanse BA, Greig NH, Mattson MP, Cader MZ, Bohr VA, et al. Mitophagy and alzheimer's disease: cellular and molecular mechanisms. Trends Neurosci. 2017;40:151-66.

20. Minibayeva F, Dmitrieva S, Ponomareva A, Ryabovol V. Oxidative stress-induced autophagy in plants: the role of mitochondria. Plant Physiol Biochem. 2012;59:11-19.

21. Zhang J, Ney PA. Role of BNIP3 and NIX in cell death, autophagy, and mitophagy. Cell Death Differ. 2009;16:939-46.

22. Palikaras K, Lionaki E, Tavernarakis N. Coordination of mitophagy and mitochondrial biogenesis during ageing in C. elegans. Nature. 2015;521:525-8.

23. Georgakopoulos ND, Wells G, Campanella M. The pharmacological regulation of cellular mitophagy. Nat Chem Biol. 2017;13:136-46.

24. Liu L, Sakakibara K, Chen Q, Okamoto K. Receptor-mediated mitophagy in yeast and mammalian systems. Cell Res. 2014;24:787-95.

25. Agostini M, Almeida LY, Bastos DC, Ortega RM, Moreira FS, Seguin F, et al. The fatty acid synthase inhibitor orlistat reduces the growth and metastasis of orthotopic tongue oral squamous cell carcinomas. Mol Cancer Ther. 2014;13:585-95.

26. Zhou Y, Yang G, Tian H, Hu Y, Wu S, Geng Y, et al. Sulforaphane metabolites cause apoptosis via microtubule disruption in cancer. Endocr Relat Cancer. 2018;25:255-68. 
27. Sirajuddin M, Rice LM, Vale RD. Regulation of microtubule motors by tubulin isotypes and post-translational modifications. Nat Cell Biol. 2014;16:335-44.

28. Lopez-Domenech G, Covill-Cooke C, Ivankovic D, Halff EF, Sheehan DF, Norkett R, et al. Miro proteins coordinate microtubule- and actin-dependent mitochondrial transport and distribution. EMBO J. 2018;37:321-36.

29. Liao S, Rajendraprasad G, Wang N, Eibes S, Gao J, Yu H, et al. Molecular basis of vasohibins-mediated detyrosination and its impact on spindle function and mitosis. Cell Res. 2019;29:533-47.

30. Menendez JA, Lupu R. Fatty acid synthase and the lipogenic phenotype in cancer pathogenesis. Nat Rev Cancer. 2007;7:763-77.

31. Jafari N, Drury J, Morris AJ, Onono FO, Stevens PD, Gao T, et al. De novo fatty acid synthesis-driven sphingolipid metabolism promotes metastatic potential of colorectal cancer. Mol Cancer Res. 2019;17:140-52.

32. Vyas AR, Hahm ER, Arlotti JA, Watkins S, Stolz DB, Desai D, et al. Chemoprevention of prostate cancer by d,l-sulforaphane is augmented by pharmacological inhibition of autophagy. Cancer Res. 2013;73:5985-95.

33. Cipolla BG, Mandron E, Lefort JM, Coadou Y, Della Negra E, Corbel L, et al. Effect of sulforaphane in men with biochemical recurrence after radical prostatectomy. Cancer Prev Res (Philos). 2015;8:712-9.

34. Williams KJ, Argus JP, Zhu Y, Wilks MQ, Marbois BN, York AG, et al. An essential requirement for the SCAP/SREBP signaling axis to protect cancer cells from lipotoxicity. Cancer Res. 2013;73:2850-62.

35. Rayner KJ, Esau CC, Hussain FN, McDaniel AL, Marshall SM, van Gils JM, et al. Inhibition of miR-33a/b in non-human primates raises plasma HDL and lowers VLDL triglycerides. Nature. 2011;478:404-7.

36. Galbraith L, Leung HY, Ahmad I. Lipid pathway deregulation in advanced prostate cancer. Pharm Res. 2018;131:177-84.

37. Chen C, Han X, Zou X, Li Y, Yang L, Cao K, et al. 4-methylene-2-octyl-5-oxotetrahydrofuran-3-carboxylic acid (C75), an inhibitor of fatty-acid synthase, suppresses the mitochondrial fatty acid synthesis pathway and impairs mitochondrial function. J Biol Chem. 2014;289:17184-94.

38. Dong H, Czaja MJ. Regulation of lipid droplets by autophagy. Trends Endocrinol Metab. 2011;22:234-40.

39. Li CF, Fang FM, Chen YY, Liu TT, Chan TC, Yu SC, et al. Overexpressed fatty acid synthase in gastrointestinal stromal tumors: targeting a progression-associated metabolic driver enhances the antitumor effect of imatinib. Clin Cancer Res. 2017;23:4908-18.

40. Grube S, Dunisch P, Freitag D, Klausnitzer M, Sakr Y, Walter J, et al. Overexpression of fatty acid synthase in human gliomas correlates with the WHO tumor grade and inhibition with Orlistat reduces cell viability and triggers apoptosis. J Neurooncol. 2014;118:277-87.

41. Hsu P, Liu X, Zhang J, Wang HG, Ye JM, Shi Y. Cardiolipin remodeling by TAZ/ tafazzin is selectively required for the initiation of mitophagy. Autophagy. 2015;11:643-52.

42. Chu CT, Ji J, Dagda RK, Jiang JF, Tyurina YY, Kapralov AA, et al. Cardiolipin externalization to the outer mitochondrial membrane acts as an elimination signal for mitophagy in neuronal cells. Nat Cell Biol. 2013;15:1197-205.

43. Hsu P, Shi Y. Regulation of autophagy by mitochondrial phospholipids in health and diseases. Biochim Biophys Acta Mol Cell Biol Lipids. 2017;1862:114-29.

44. Chourasia AH, Macleod KF. Tumor suppressor functions of BNIP3 and mitophagy. Autophagy. 2015;11:1937-8.

45. Rodger CE, McWilliams TG, Ganley IG. Mammalian mitophagy - from in vitro molecules to in vivo models. FEBS J. 2018;285:1185-202.

46. Esteban-Martinez L, Boya P. BNIP3L/NIX-dependent mitophagy regulates cell differentiation via metabolic reprogramming. Autophagy. 2018;14:915-7.

47. Ding WX, Ni HM, Li M, Liao Y, Chen X, Stolz DB, et al. Nix is critical to two distinct phases of mitophagy, reactive oxygen species-mediated autophagy induction and Parkin-ubiquitin-p62-mediated mitochondrial priming. $J$ Biol Chem. 2010;285:27879-90.

48. Sandoval H, Thiagarajan P, Dasgupta SK, Schumacher A, Prchal JT, Chen M, et al. Essential role for Nix in autophagic maturation of erythroid cells. Nature. 2008:454:232-5.

49. Zeng X, Li Y, Fan J, Zhao H, Xian Z, Sun Y, et al. Recombinant human arginase induced caspase-dependent apoptosis and autophagy in non-Hodgkin's lymphoma cells. Cell Death Dis. 2013;4:e840.

50. Palikaras K, Lionaki E, Tavernarakis N. Mechanisms of mitophagy in cellular homeostasis, physiology and pathology. Nat Cell Biol. 2018;20:1013-22.
51. Onorati AV, Dyczynski M, Ojha R, Amaravadi RK. Targeting autophagy in cancer Cancer. 2018;124:3307-18.

52. Zheng Z, Lin K, Hu Y, Zhou Y, Ding X, Wang Y, et al. Sulforaphane metabolites inhibit migration and invasion via microtubule-mediated Claudins dysfunction or inhibition of autolysosome formation in human non-small cell lung cancer cells. Cell Death Dis. 2019:10:259.

\section{ACKNOWLEDGEMENTS}

We thank Zhongxin Xiao at the Facility Center of Capital Medical University for help in immunofluorescence confocal assay. All authors participated in the experiments and reviewed the manuscript.

\section{AUTHOR CONTRIBUTIONS}

W.W. conceived, designed, and supervised the entire project. W.W., Y.T.Y., and Y.Z. developed the methodology. Y.T.Y., Y.Z., J.T.L., and Z.N.Z. acquired the data. Y.Z., Y.T. Y., Y.B.H., and Z.N.Z. analyzed and interpreted the data (e.g., statistical analysis and computational analysis). W.W., Y.T.Y., and Y.Z. wrote and revised the manuscript. T.Z. Z.N.Z., J.T.L., and L.L. provided administrative and technical support (i.e., organizing data and constructing databases)

\section{FUNDING}

The present study was supported by the National Natural Science Foundation of China (grant numbers 81272843 and 81601993 ) and Support Project of High-level Teachers in Beijing Municipal Universities in the Period of 13th Five-Year Plan (grant number IDHT20190510).

\section{ETHICS STATEMENT}

This study is not involved in ethics.

\section{COMPETING INTERESTS}

The authors declare no competing interests.

\section{ADDITIONAL INFORMATION}

Correspondence and requests for materials should be addressed to Wei Wu.

Reprints and permission information is available at http://www.nature.com/ reprints

Publisher's note Springer Nature remains neutral with regard to jurisdictional claims in published maps and institutional affiliations.

Open Access This article is licensed under a Creative Commons Attribution 4.0 International License, which permits use, sharing, adaptation, distribution and reproduction in any medium or format, as long as you give appropriate credit to the original author(s) and the source, provide a link to the Creative Commons license, and indicate if changes were made. The images or other third party material in this article are included in the article's Creative Commons license, unless indicated otherwise in a credit line to the material. If material is not included in the article's Creative Commons license and your intended use is not permitted by statutory regulation or exceeds the permitted use, you will need to obtain permission directly from the copyright holder. To view a copy of this license, visit http://creativecommons. org/licenses/by/4.0/.

(c) The Author(s) 2021 